\title{
Next-to-leading order QCD corrections to di-photon production in association with up to three jets at the Large Hadron Collider
}

\author{
Simon Badger, ${ }^{a}$ Alberto Guffanti ${ }^{b}$ and Valery Yundin ${ }^{c}$ \\ ${ }^{a}$ Theory Division, Physics Department, CERN, \\ CH-1211 Geneva 23, Switzerland \\ ${ }^{b}$ Niels Bohr International Academy and Discovery Center, The Niels Bohr Institute, \\ University of Copenhagen, Blegdamsvej 17, DK-2100 Copenhagen, Denmark \\ ${ }^{c}$ Max-Planck-Institut für Physik, \\ Föhringer Ring 6, 80805 Munich, Germany \\ E-mail: simon.badger@cern.ch, alberto.guffanti@nbi.dk, \\ yundin@mpp.mpg.de
}

ABSTRACT: We present the computation of next-to-leading order (NLO) QCD corrections to di-photon production in association with two or three hard jets in $p p$ collisions at a centre-of-mass energy of $8 \mathrm{TeV}$. The inclusion of NLO corrections is shown to substantially reduce the theoretical uncertainties estimated from scale variations on total cross section predictions. We study a range of differential distributions relevant for phenomenological studies of photon pair production in association with jets at the LHC. Using an efficient computational set-up we performed a detailed study of uncertainties due to parton distribution functions. The computation of the virtual corrections is performed using new features of the $\mathrm{C}++$ library NJET.

Keywords: NLO Computations, Jets

ARXIV EPRINT: 1312.5927 


\section{Contents}

1 Introduction 1

2 Computational set-up 2

3 Numerical results 4

$3.1 \quad$ Results for $p p \rightarrow \gamma \gamma+2 j$

3.2 Results for $p p \rightarrow \gamma \gamma+3 j \quad 9$

$\begin{array}{ll}3.3 & \text { The three-to-two jet ratio } \\ \end{array}$

4 Conclusions 13

\section{Introduction}

There has been a lot of recent interest in the study of $p p \rightarrow \gamma \gamma+$ jets processes as an important background to $p p \rightarrow H \rightarrow \gamma \gamma$ which is one of the cleanest decay channels for studies of the Higgs properties [1, 2]. These processes also have importance outside the realm of Higgs physics testing our ability to model isolated photon radiation in association with strong interactions, see for example refs. [3, 4] for recent experimental studies of the closely related process of photon production in association with hard jets. From a theoretical point of view di-photon production is under good control with corrections known up to NNLO in QCD [5]. NLO QCD corrections to $p p \rightarrow \gamma \gamma+j$ have been available for some time [6] and have recently been re-explored [7] to investigate the impact of using different photon isolation criteria comparing the smooth cone isolation [8] with the fixed cone isolation favoured in experimental studies. The computation of $p p \rightarrow \gamma \gamma+2 j$ including NLO QCD corrections has been completed quite recently $[9,10]$.

In this paper we extend the available range of predictions for $p p \rightarrow \gamma \gamma+$ jets to include up to three hard jets with NLO accuracy in QCD. The results show a significant reduction in the uncertainty of the theoretical predictions and have noticeable corrections to the shapes of the distributions when going from LO to NLO. As well as providing new phenomenological studies relevant for the current measurements at ATLAS and CMS we also present new developments to the NJET C ++ code enabling more efficient computations of high multiplicity processes at NLO.

Modern methods for scattering amplitude computations based on unitarity [11], generalized unitarity [12] and integrand reduction [13] have opened up the possibility of performing precision phenomenological studies with multi-particle final states at colliders. The current state-of-the-art NLO QCD processes include $p p \rightarrow W / Z+4 j[14,15], p p \rightarrow 4 j[16,17]$, $p p \rightarrow W+5 j$ [18], $p p \rightarrow 5 j$ [19], all of which have been obtained using on-shell methods. 
A wealth of $2 \rightarrow 4$ and general lower multiplicity processes are now becoming available in an increasing number automated codes [20-25].

This article is organized as follows: we begin by outlining our computational set-up in section 2, where for completeness we review the well known decomposition of nextto-leading order differential cross sections and describe the interface of NJET with the Sherpa Monte-Carlo, which we used for the computation of the unresolved real radiation contributions and phase-space integration. In section 3 we provide results for the LHC at centre-of-mass energy of $8 \mathrm{TeV}$ for both $p p \rightarrow \gamma \gamma+2 j$ and $p p \rightarrow \gamma \gamma+3 j$. We present differential distributions for some important observables, particularly those used in Higgs productions studies with vector-boson fusion phase space in the case of $p p \rightarrow \gamma \gamma+2 j$. We present a study of the dependence on the renormalization scale of the NLO predictions and investigate the uncertainty due to the choice of Parton Distribution Functions (PDFs) on total cross sections and distributions. In section 4 we present our conclusions.

\section{Computational set-up}

The computation is performed in the five-flavour scheme with massless b-quark included in the initial state. The basic partonic sub-processes considered are:

$$
0 \rightarrow \gamma \gamma q \bar{q} g g
$$

for $p p \rightarrow \gamma \gamma+2 j$ and

$$
0 \rightarrow \gamma \gamma q \bar{q} g g g \quad 0 \rightarrow \gamma \gamma q \bar{q} q^{\prime} \bar{q}^{\prime} g
$$

for $p p \rightarrow \gamma \gamma+3 j$, from which all relevant channels can be derived using crossing symmetries. Channels with like-flavour fermions are obtained from the above using the appropriate (anti-)symmetrization relations. We do not include loop-induced and formally higher order sub-processes $0 \rightarrow \gamma \gamma+4 g$ and $0 \rightarrow \gamma \gamma+5 g$.

We can schematically write down the NLO partonic cross section as a sum of four finite contributions which can be integrated separately over their respective phase spaces,

$$
d \sigma_{n}^{\mathrm{NLO}}=\int_{n} d \sigma_{n}^{B}+\int_{n} d \sigma_{n}^{V}+\int_{n} d \sigma_{n}^{I}+\int_{n+1} d \sigma_{n+1}^{R S}
$$

where $d \sigma_{n}^{B}$ denotes the leading order contribution, $d \sigma_{N}^{I}$ contains the integrated dipole subtraction terms, including factorization contributions from initial state singularities, $d \sigma_{n}^{V}$ the one-loop virtual corrections and $d \sigma_{n+1}^{R S}$ the infra-red finite contributions from realradiation and dipole subtraction terms. This hard partonic cross section is then convoluted with the parton distribution functions to obtain the cross sections for hadronic collisions.

The computation of the Born and real-emission matrix elements is performed using the colour dressed recursive Berends-Giele formulation [26] implemented in the Comix amplitude generator [27]. The subtraction of infra-red singularities is performed using the Catani-Seymour [28] dipole method. The evaluation of these contributions is performed using the Sherpa $[29,30]$ package, which is also used for the determination and organization 
of the partonic subprocesses and the integration over the phase space. The one-loop virtual amplitudes are provided using the automated generalized unitarity framework implemented in the latest version of the NJET library. ${ }^{1}$

NJET 2.0 is an updated code based on NJet [31] and NGLuON [32]. The primitive kinematic objects are constructed using a numerical generalized unitarity algorithm [1113, 20, 33-36] with Berends-Giele recursion relations for the tree-level input [26]. The extended code can compute arbitrary primitive amplitudes for vector bosons ( $\mathrm{W}, \mathrm{Z}$ and $\gamma$ ) and massless QCD partons. Full colour sums are implemented for pure QCD with up to five jets, vector bosons with up to five jets and di-photon production with up to four jets. We interface this code to the Sherpa Monte-Carlo via the updated Binoth Les Houches Accord $[37,38]$ to obtain the virtual events. In addition to the standard internal checks we have managed to check individual phase-space points for the processes used in this paper against those obtained with GoSam [23] and MAdLoop [21]. For both processes we have neglected the small effect of top quark loops in the virtual amplitudes. In the case of $p p \rightarrow \gamma \gamma+3 j$ we also neglect the contribution from vector loops where the photons couple directly to a virtual fermion loop. These corrections have been included in $p p \rightarrow \gamma \gamma+2 j$ contributing less than $0.5 \%$ of the total cross section, therefore they are expected to be negligible for $p p \rightarrow \gamma \gamma+3 j$.

For the current calculation we made use of some new features to optimise the computation time needed for the virtual corrections. Firstly we use a $\mathrm{C}++$ library Vc [39] to utilize vector capabilities of modern CPUs and gain a factor of $\sim 2$ in the computation speed. We also separate leading and sub-leading contributions in colour such that the simpler, dominant contributions can be sampled more often than the sub-leading terms. The definition of our leading terms include all multi-quark processes in the large $N_{c}$ limit and processes with two or more gluons in the final state using the de-symmetrized form of the colour sum that efficiently exploits the Bose symmetry of the phase space [31, 40]. The de-symmetrized sums give full colour information and are faster than leading colour when we have many final state gluons. In figure 1 we show virtual corrections to the $3 \mathrm{rd}$ leading jet transverse momentum in $p p \rightarrow \gamma \gamma+3 j$. The corrections from the sub-leading part are around $10 \%$ on average with a slight rise to around $20 \%$ at large $p_{T}$. In the case of $p p \rightarrow \gamma \gamma+3 j$ the virtual cross sections are about $1 / 3$ of the size of the total cross section. For the current implementation of $p p \rightarrow \gamma \gamma+3 j$, the leading virtual events are generated approximately 7 times faster than the sub-leading events.

In order to maximise the phenomenological predictions that we can extract from processes with such complicated final states, we make use of the ROOT Ntuple format provided by Sherpa [41]. During the course of event generation, additional weights from the poles in the loop process and from the subtraction terms are stored along with a full list of kinematic variables and couplings. This allows the events to be re-weighted to different scale and PDF choices during analysis. Even within this approach a full study of PDF uncertainties and scale variations can be computationally intensive. During this work we have developed an interface to the APPLgrid library [42] to allow for specific observables to be efficiently re-weighted changing the scales and the specific PDF set used.

\footnotetext{
${ }^{1}$ The code NJET 2.0 is available at https://bitbucket.org/njet/njet/downloads.
} 


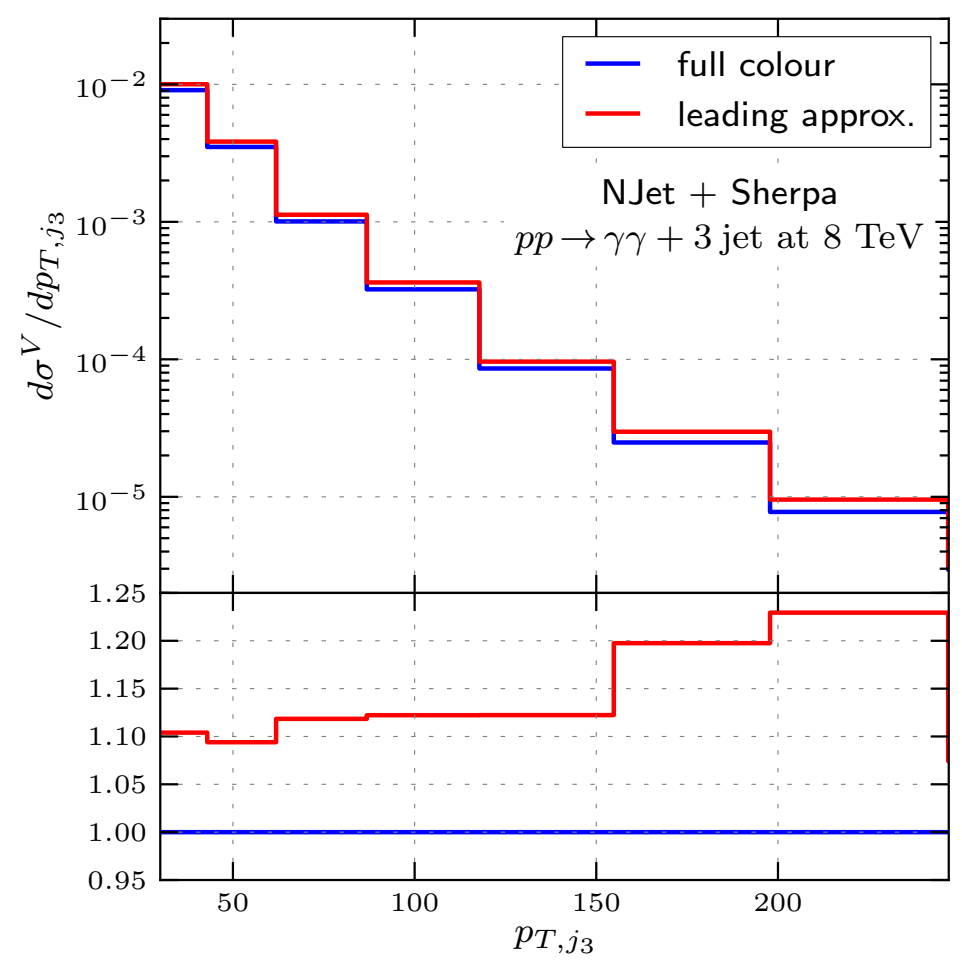

Figure 1. Full colour and leading approximation (as explained in the text) for the virtual corrections to the transverse momentum of the 3 rd jet in $p p \rightarrow \gamma \gamma+3 j$.

\section{Numerical results}

All the results presented in this section are for $p p$ collisions with a centre-of-mass energy of $8 \mathrm{TeV}$. We consider the following kinematic cuts on the external momenta, which are inspired by typical experimental cuts used in the analyses at LHC

$$
\begin{array}{lll}
p_{T, j}>30 \mathrm{GeV} & \left|\eta_{j}\right| \leq 4.7 & \\
p_{T, \gamma_{1}}>40 \mathrm{GeV} & p_{T, \gamma_{2}}>25 \mathrm{GeV} & \left|\eta_{\gamma}\right| \leq 2.5 \\
R_{\gamma, j}=0.5 & R_{\gamma, \gamma}=0.45 &
\end{array}
$$

where the photon transverse momenta have been ordered by size. The jets are defined using the anti- $k_{T}$ algorithm [43] with cone size $R=0.5$ as implemented in FASTJET [44]. Photons are selected using the Frixione smooth cone isolation criterion [8]. A photon is considered isolated if the total hadronic energy inside all cones of radius $r_{\gamma}<R$

$$
E_{\text {hadronic }}\left(r_{\gamma}\right) \leq \epsilon p_{T, \gamma}\left(\frac{1-\cos r_{\gamma}}{1-\cos R}\right)^{n}
$$

with $\epsilon=0.05, R=0.4$ and $n=1$. We use the NLO CT10 PDF set [45] for our central predictions with the strong coupling running from $\alpha_{s}\left(M_{Z}\right)=0.118$, and the electromagnetic coupling fixed at $\alpha=1 / 137.036$. In particular we use the same (NLO) PDF set and definition of the strong coupling constant both for LO and NLO predictions. Using a NLO 
PDF set for the LO computation includes higher order terms that go beyond a fixed order prediction, nevertheless such a set-up allows us to separate NLO effects coming from the running of the strong coupling and from PDFs and to highlight the impact of corrections coming from the NLO matrix elements.

We choose a dynamical value for the factorization and renormalization scales which are kept equal, $\mu_{R}=\mu_{F}$, when performing scale variations. We have investigated the dependence on a number of different functional forms which we will denote as:

$$
\begin{aligned}
\widehat{H}_{T} & =p_{T, \gamma_{1}}+p_{T, \gamma_{2}}+\sum_{i \in \text { partons }} p_{T, i} \\
\widehat{H}_{T}^{\prime} & =m_{\gamma \gamma}+\sum_{i \in \text { partons }} p_{T, i} \\
\widehat{\Sigma}^{2} & =m_{\gamma \gamma}^{2}+\sum_{i \in \text { partons }} p_{T, i}^{2} \\
H_{T}^{\prime} & =m_{\gamma \gamma}+\sum_{i \in \text { jets }} p_{T, i} \\
\Sigma^{2} & =m_{\gamma \gamma}^{2}+\sum_{i \in \text { jets }} p_{T, i}^{2}
\end{aligned}
$$

where $m_{\gamma \gamma}=\sqrt{\left(p_{\gamma_{1}}+p_{\gamma_{2}}\right)^{2}}$. The quantities $H_{T}^{\prime}$ and $\Sigma^{2}$ are constructed after the clustering of final state partons into jets. Notice that partonic and jet-level scales will only differ at NLO, where the additional unresolved radiation enters in the clustering algorithm.

\subsection{Results for $p p \rightarrow \gamma \gamma+2 j$}

We first consider the production of a photon pair in association with two jets. In this case we compare our predictions with the recent results of reference [9] and present additional studies of PDF variations and dynamical scale choices. For the latter we rely on the possibility to substantially reduce the computational cost by the use of our APPLgrid set-up.

In figure 2 we show the dependence of the total inclusive cross section upon variation of the renormalization and factorization scales with $\mu_{R}=\mu_{F}$. We consider the five different dynamical scales defined in eq. (3.6). Though the scale choices are closely related to each other we find significant deviations for the value of the total cross section. Nevertheless the NLO predictions show a significant reduction in the dependence on the scale variation compared to the LO ones. Taking the envelope of all the scales considered we see that the LO predictions vary in the interval $1.64-3.04$ whereas NLO ones lie within 2.46-3.58 when the scales are varied over the range $x \in[0.5,2]$ around the central choice. This represents a reduction in the scale variation uncertainty from $\sim 30 \%$ at LO to $\sim 20 \%$ at NLO.

We notice that choosing $\mu_{R}=\sqrt{\widehat{\Sigma}^{2}}$ as a scale leads to significantly larger predictions for the total cross section than with the other scales considered here. Moreover the scale variation profile closely resembles the one of a leading order prediction. We therefore consider this choice of scale disfavoured with respect to the others, which provide results that are in better agreement with each other. In general, we find that the $H_{T}$-based scales lead to a broader profile of scale variations and on average favour harder events than the 


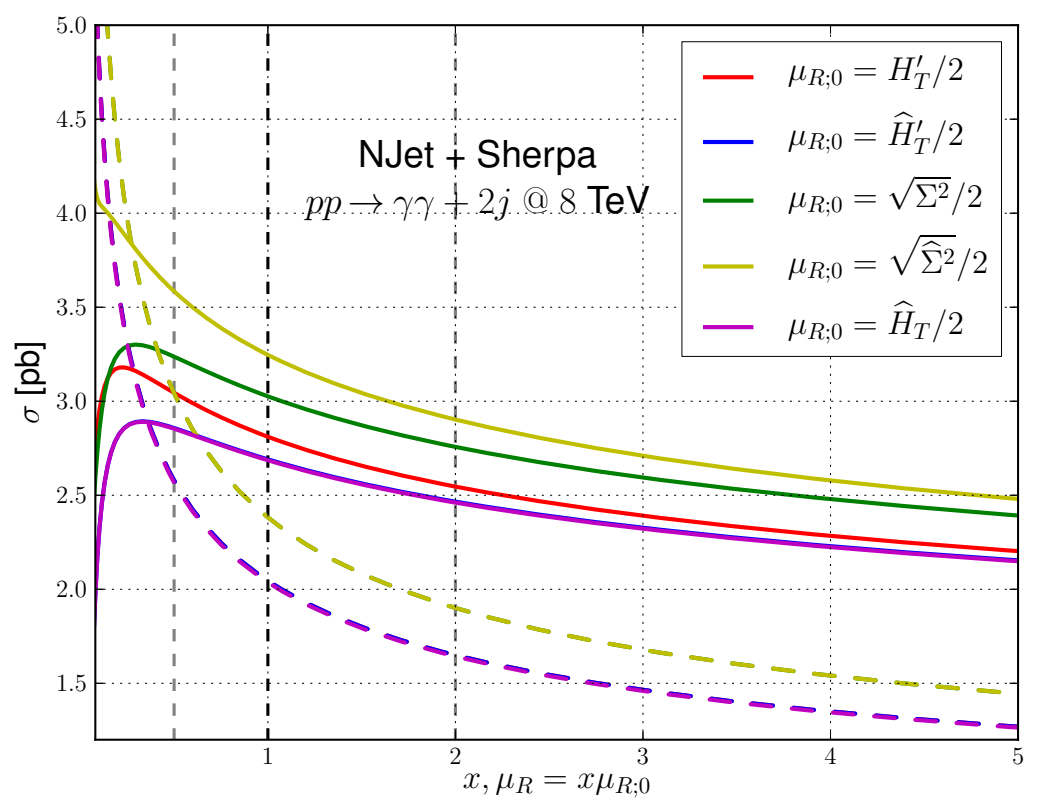

Figure 2. Scale variations on the total cross section for $p p \rightarrow \gamma \gamma+2 j$ for a variety of dynamical scales. Dashed lines are LO accuracy whereas solid lines are NLO accuracy. The vertical line at $x=1$ corresponds to the central scale whereas the lines at $x=0.5$ and $x=2$ show the bounds of the scale variation region.

$\Sigma$-based scales. On the other hand we find the shapes of the distributions to be quite stable with respect to the scale choice.

The results for the total cross section and distributions using $\mu_{R}=\sqrt{\Sigma^{2}} / 2$ are in good agreement with those obtained previously by Gehrmann, Greiner and Heinrich [9]. In the following we opt for the scale $\widehat{H}_{T}^{\prime}$ for computing our predictions for the total cross section and the differential distributions presented here. This scale has been widely used in studies of $W+$ jets (see for example [18]).

The values for the total cross sections at both LO and NLO computed our default choices for scale, PDF set and physical parameters are found to be:

$$
\sigma_{\gamma \gamma+2 j}^{\mathrm{LO}}\left(\widehat{H}_{T}^{\prime} / 2\right)=2.046(0.002)_{-0.396}^{+0.534} \mathrm{pb} \quad \sigma_{\gamma \gamma+2 j}^{\mathrm{NLO}}\left(\widehat{H}_{T}^{\prime} / 2\right)=2.691(0.007)_{-0.225}^{+0.167} \mathrm{pb}
$$

where the sub-scripts(super-scripts) show the maximum deviation from the central value in the negative(positive) direction over the range $x \in[0.5,2]$ for $\mu_{R}=x \widehat{H}_{T}^{\prime} / 2$. Monte-Carlo integration errors are shown in brackets.

Figure 3 shows the differential distributions for the ordered jet transverse momenta. The results for the scale $\widehat{H}_{T}^{\prime} / 2$ are consistent with those obtained at the scale $\sqrt{\Sigma^{2}}$ with a significant reduction in scale variation from around $20 \%$ at LO to $\sim 10 \%$ at NLO in both cases. The $K$-factor is fairly constant at around 1.1 for $p_{T}$ higher than $200 \mathrm{GeV}$ rising to 1.4 as we approach the $p_{T}$ cut. This larger $K$-factor in the low $p_{T}$ region could be the indication of the presence of large logarithms beyond fixed order NLO. The di-photon invariant mass $m_{\gamma \gamma}$ and the di-photon rapidity distributions $\eta_{\gamma \gamma}$ are shown in figure 4 . 

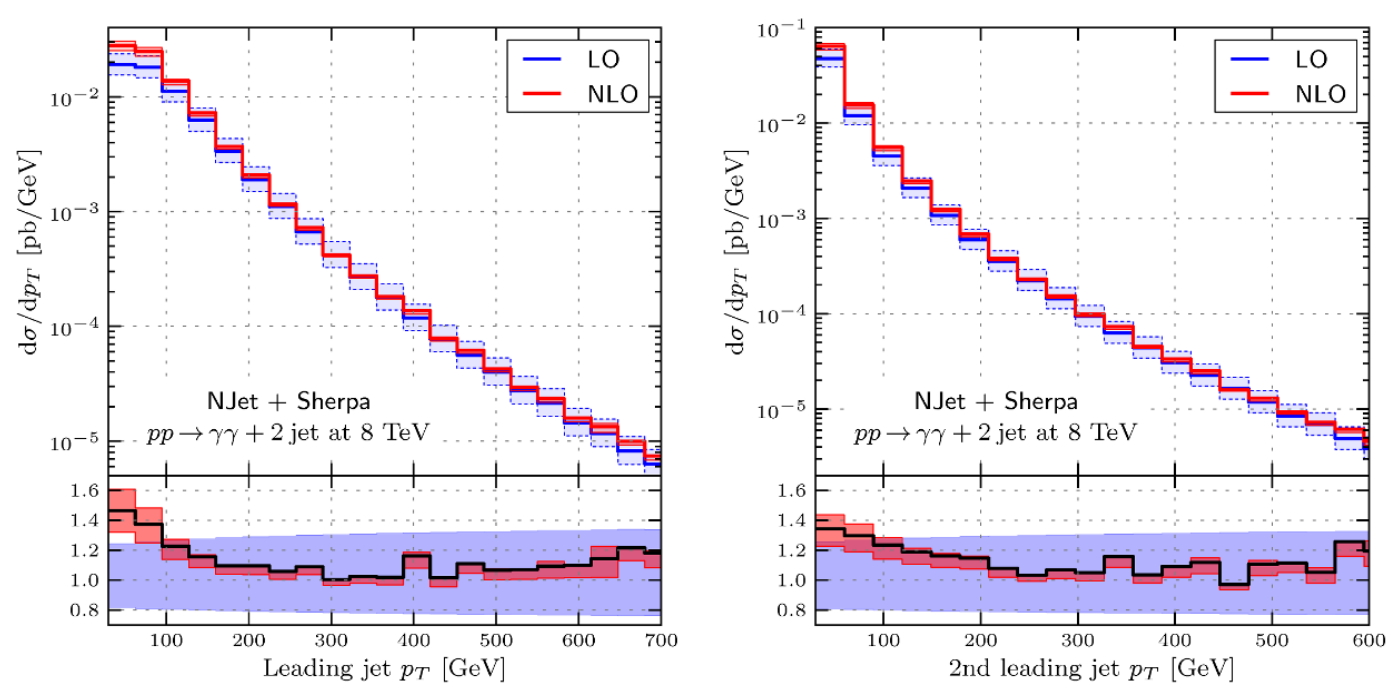

Figure 3. Differential distributions for jet transverse momenta. The lower plot shows the ratio of NLO to LO including the scale variation bands estimated over the range of $x \in[0.5,2]$.

They receive slightly larger NLO corrections with respect to the jet transverse momenta with the $K$-factor for the former ranging from 1.2 in the large invariant mass region to 1.7 for lower invariant masses, while for the latter NLO correction induce a roughly flat $K$-factor of 1.3 .

Figure 5 shows four distributions of angular quantities that are usually used in analyses of Higgs production in vector boson fusion (VBF), where additional cuts are imposed in order to reduce QCD backgrounds in $p p \rightarrow H(\rightarrow \gamma \gamma)+2 j$ studies.

Owing to the increased phase-space available to the real radiation at NLO we see large deviations from the shapes of the leading order distributions. These features have been pointed out for the jet-pair azimuthal angle $\Delta \phi_{j_{1} j_{2}}$ and for the separation of the leading-photon/leading jet,

$$
R_{\gamma_{1} j_{1}}=\sqrt{\Delta y_{\gamma_{1} j_{1}}^{2}+\Delta \phi_{\gamma_{1} j_{1}}^{2}}
$$

in ref. [9]. We also see increasing deviations for large rapidities of the jet pair $\eta_{j_{1} j_{2}}$ and even more so in the relative rapidity of the diphoton-dijet system,

$$
y_{\gamma \gamma j j}^{*}=y_{\gamma \gamma}-\left(y_{j_{1}}+y_{j_{2}}\right) / 2 .
$$

Using the APPLgrid set-up described in the previous section, we have also performed a study of PDF uncertainties on $p p \rightarrow \gamma \gamma+2 j$, concentrating on the total cross section and the invariant mass distribution of the photon pair. In table 1 we show the central value and PDF uncertainties for the total cross section evaluated at the central scale $\left(\mu_{R}=\right.$ $\left.\widehat{H}_{T}^{\prime} / 2\right)$ using four different NLO PDF sets: CT10 [45], MSTW2008 [46], ABM11 [47] and NNPDF2.3 [48]. All PDF sets are compared using the same value of $\alpha_{s}\left(M_{Z}\right)=0.118$ in order to disentangle PDF and strong coupling constant uncertainties. PDF uncertainties are considerably smaller than the theoretical uncertainty estimated from scale variations 

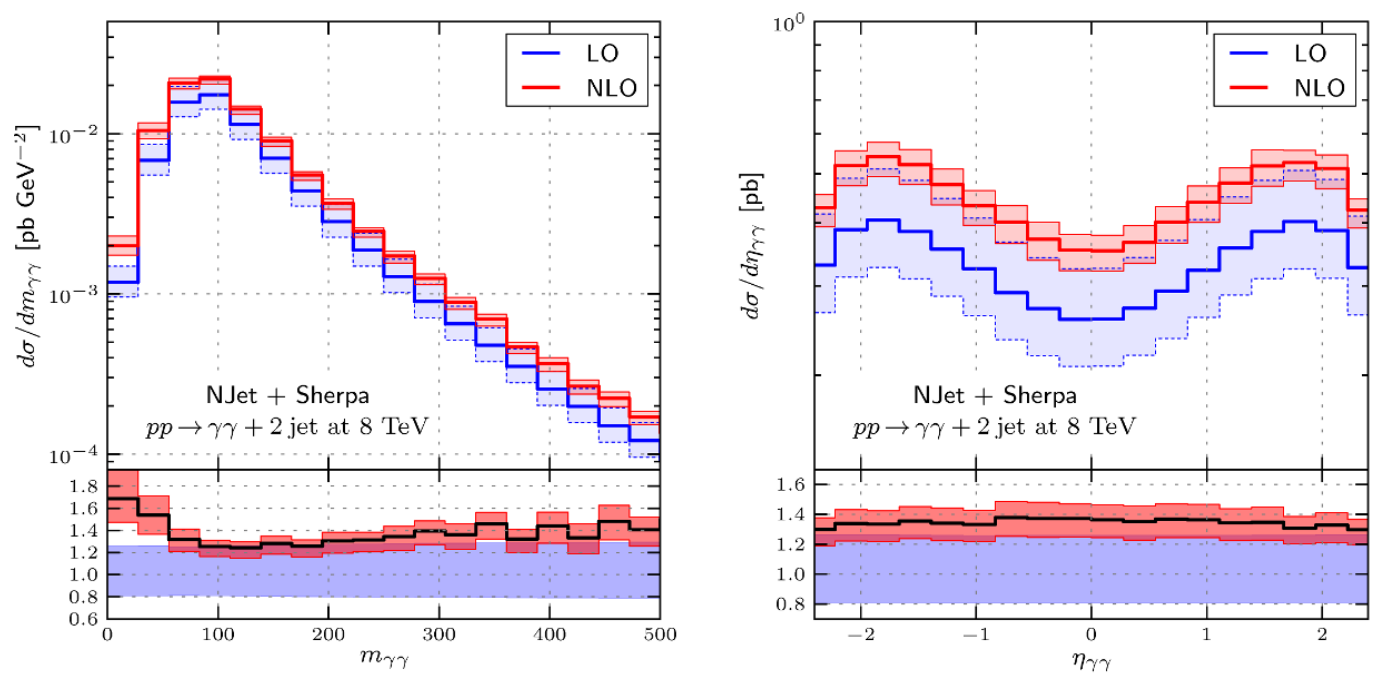

Figure 4. Differential distributions for di-photon invariant mass and rapidity $p p \rightarrow \gamma \gamma+2 j$.

\begin{tabular}{|lccc|}
\hline PDF set & $\sigma_{\gamma \gamma+2 j}^{\mathrm{NLO}}\left(\widehat{H}_{T}^{\prime} / 2\right)$ & $\delta \sigma_{\gamma \gamma+2 j}^{\mathrm{NLO}, \mathrm{PDF}+}\left(\widehat{H}_{T}^{\prime} / 2\right)$ & $\delta \sigma_{\gamma \gamma+2 j}^{\mathrm{NLO}, \mathrm{PDF}-}\left(\widehat{H}_{T}^{\prime} / 2\right)$ \\
\hline CT10nlo & 2.69102 & +0.0357456 & -0.042148 \\
NNPDF2.3 & 2.77285 & +0.0167702 & -0.016770 \\
MSTW2008 & 2.71578 & +0.0184072 & -0.016373 \\
ABM11 & 2.73791 & +0.0659662 & -0.065966 \\
\hline
\end{tabular}

Table 1. The total cross section for $p p \rightarrow \gamma \gamma+2 j$ computed with different PDF sets at $\alpha_{s}\left(M_{Z}\right)=$ 0.118. The PDF uncertainties are computed from the relevant PDF error sets.

and range from $1 \%$ for MSTW and NNPDF to $3.5 \%$ for ABM. We note, however that the ABM11 uncertainty includes errors associated with $\alpha_{s}$ variations. In general, we find that central predictions from different PDF sets differ by amounts which are larger than the nominal PDF uncertainty of each set.

In figure 6 we show the distributions for the invariant mass of the photon pair $\left(m_{\gamma \gamma}\right)$ computed at the scale $\mu_{R}=\widehat{H}_{T}^{\prime} / 2$ and the four PDF sets considered before at $\alpha_{s}\left(M_{Z}\right)=$ 0.118. The upper (log scale) plot shows the absolute predictions for all sets, which are indeed extremely close to each other. In the lower plot, where we show the ratio of each set to the central value computed using CT10, we do however notice discrepancies in the predictions of the order of a few percent. These differences are especially pronounced at high $m_{\gamma \gamma}$ where the spread between them reaches $\sim 6 \%$, a value larger than PDF uncertainties coming from individual sets (represented by the shaded regions). We also note that, while the differences between CT10, MSTW08 and NNPDF2.3 are mostly due to an overall normalization factor, the ABM11 predictions stand out from the others also in the shape being higher for low values of $m_{\gamma \gamma}$ and dropping below the other predictions for higher values of the invariant mass. 

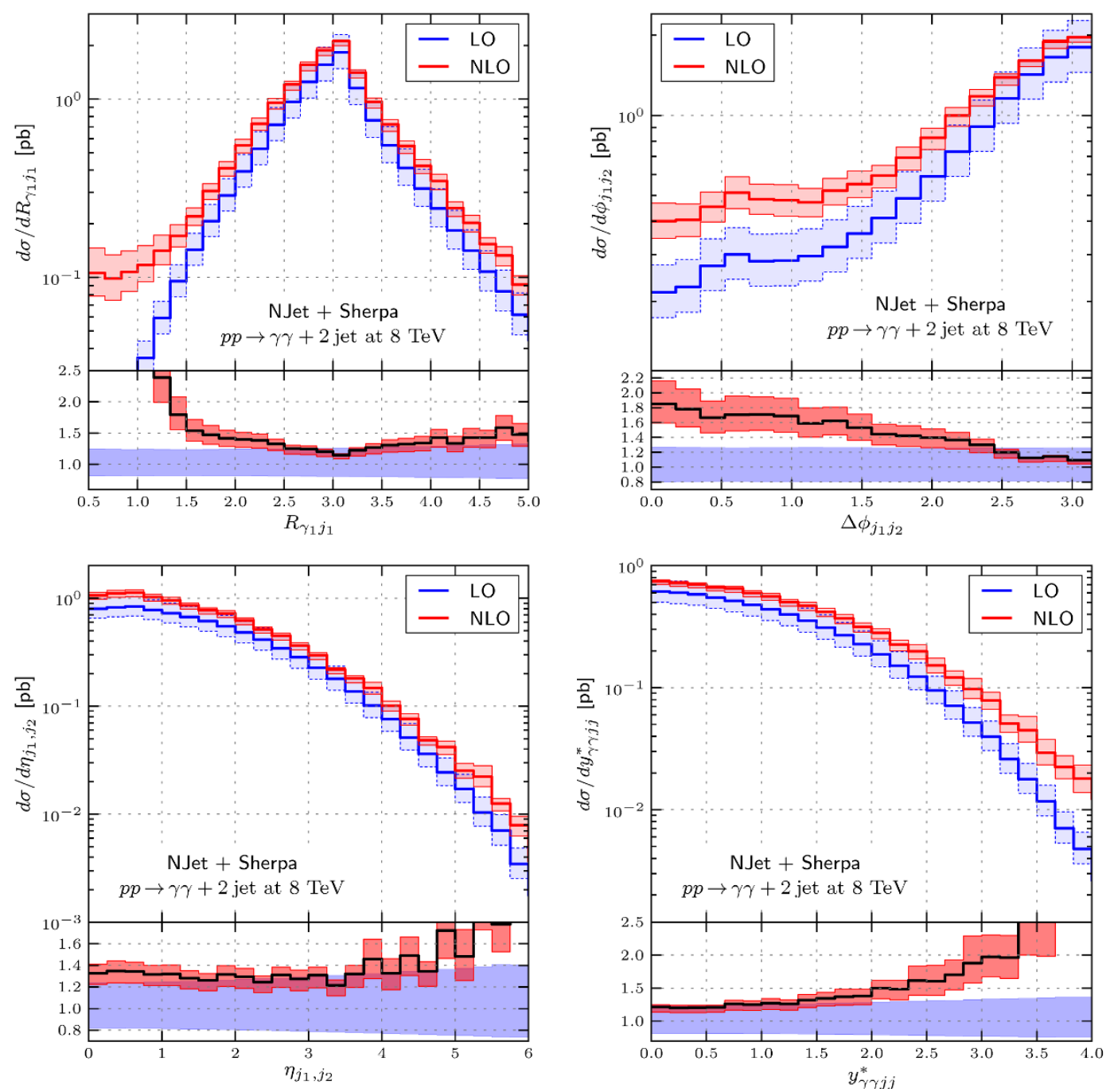

Figure 5. Differential distributions for the angular observables $R_{\gamma_{1} j_{1}}$ (see eq. (3.8)), $\Delta \phi_{j_{1} j_{2}}, \eta_{j_{1} j_{2}}$ and $y_{\gamma \gamma j j}^{*}$ (see eq. (3.9)) in $p p \rightarrow \gamma \gamma+2 j$.

\section{$3.2 \quad$ Results for $p p \rightarrow \gamma \gamma+3 j$}

We now consider the production of a photon pair in association with three jets. As in the previous section we studied the dependence of the total cross section upon variation of renormalization and factorization scales with the choices of dynamical scales defined in eq. (3.6). The results in figure 7 show reasonable differences between quantities based on jets versus quantities based on partons. Overall we find a significant improvement in the uncertainty estimated from scale variations when going from LO to NLO. The envelope of predictions from all scale choices varied over the range $x \in[0.5,2]$ is around $0.67-0.99 \mathrm{pb}$ at NLO compared to $0.46-1.28 \mathrm{pb}$ at LO. This represents a decrease in variation from $\sim 50 \%$ at LO to $\sim 20 \%$ at NLO. As in the two jet case the scales based on $\Sigma^{2}$ give generally larger predictions than those based on $H_{T}$. Other than the overall normalization, we find that all scales give very similar predictions for shapes of the distributions. 


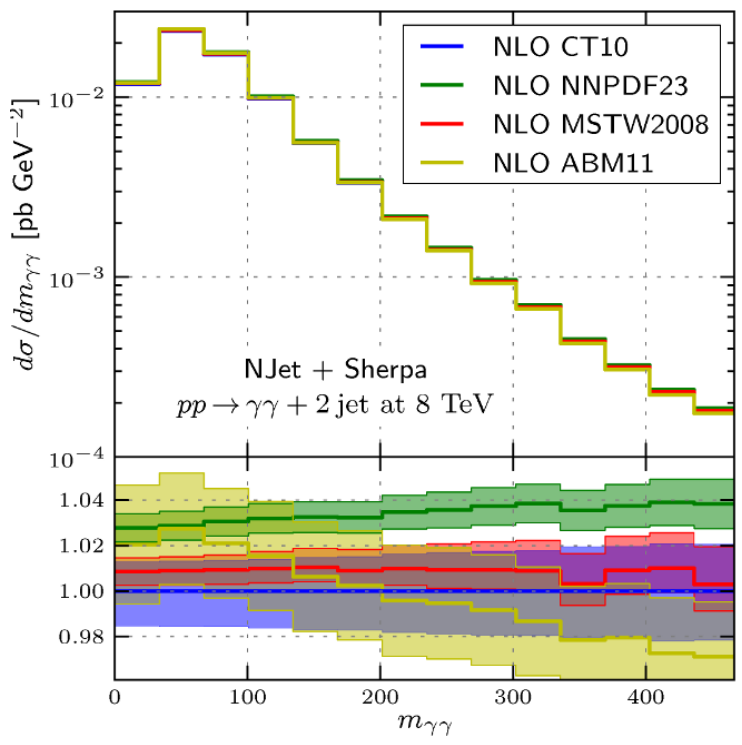

Figure 6. The $m_{\gamma \gamma}$ distributions for $p p \rightarrow \gamma \gamma+2 j$ for the four PDF sets described in the text at $\alpha_{s} M_{Z}=0.118$. The lower plot shows the ratio of each set to CT10 with the shaded region representing the PDF uncertainty.

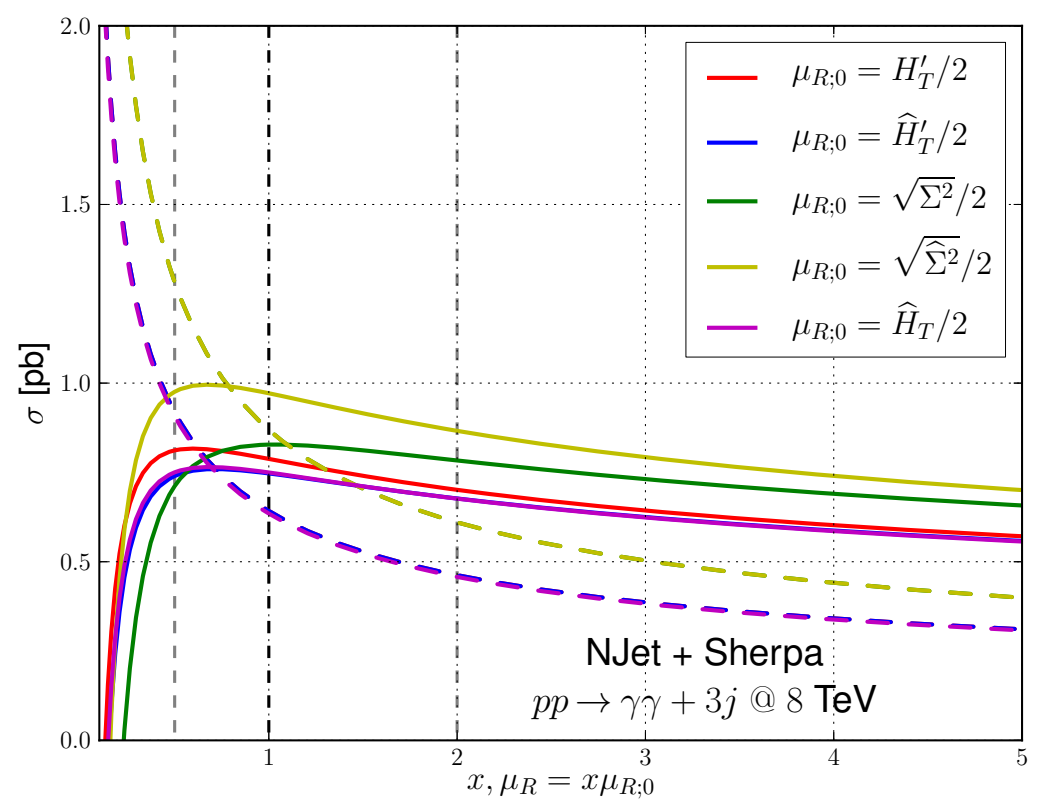

Figure 7. The result of scale variations on the total inclusive cross section for $p p \rightarrow \gamma \gamma+3 j$. LO curves are represented with dashed lines while NLO curves are represented with solid lines.

Comparing figure 7 with figure 2 we see that the peak in the NLO curve for $\Sigma^{2}$ has moved further to the right than the $H_{T}$ scales which may suggest that a range of $x \in[1,4]$ would be more appropriate here. Since we would like to make predictions for jet ratios we need to have as consistent description of $\gamma \gamma+3 j$ and $\gamma \gamma+2 j$ as possible and therefore we prefer the $H_{T}$ scales. In the following we choose to adopt the central scale of $\widehat{H}_{T}^{\prime} / 2$ for the 

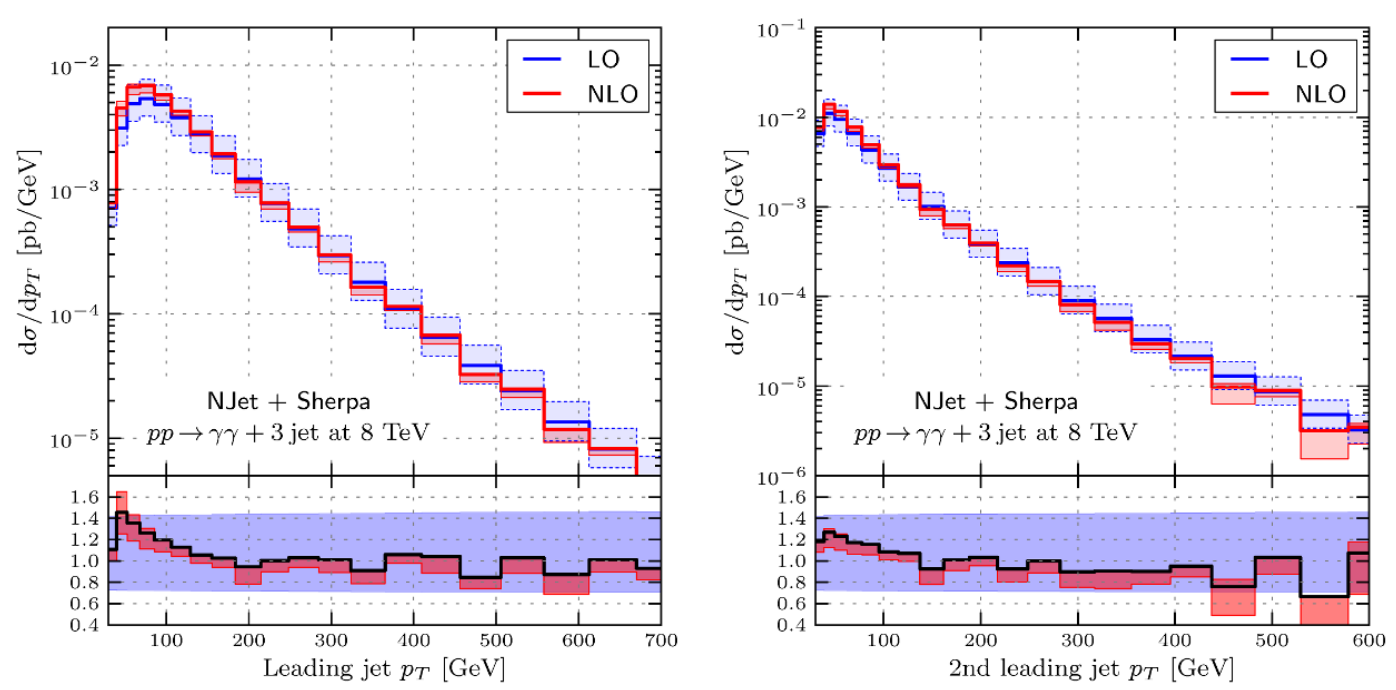

Figure 8. Differential cross section as a function of $p_{T}$ of the 1 st and 2 nd leading jets.

total rates and distributions, though theoretical uncertainties are likely underestimated by the simple scale variations following the discussion above. For the total cross sections at LO and NLO we find,

$$
\sigma_{\gamma \gamma+3 j}^{\mathrm{LO}}\left(\widehat{H}_{T}^{\prime} / 2\right)=0.643(0.003)_{-0.180}^{+0.278} \mathrm{pb} \quad \sigma_{\gamma \gamma+3 j}^{\mathrm{NLO}}\left(\widehat{H}_{T}^{\prime} / 2\right)=0.785(0.010)_{-0.085}^{+0.027} \mathrm{pb}
$$

where the sub-scripts(super-scripts) show the maximum deviation from the central value in the negative(positive) direction over the range $x \in[0.5,2]$ for $\mu_{R}=x \widehat{H}_{T}^{\prime} / 2$ and MonteCarlo integration errors are shown in brackets.

The distributions for the jets transverse momenta are shown in figure 8 . The $K$-factor is quite flat with a value between 1.0 and 1.2 except in the low $p_{T}$ where it rises to around 1.4 for the leading jet $p_{T}$. Again, this may suggest the presence of large logarithms in the missing higher order contributions in this region. The distribution of the 3rd jet shown in figure 9 (a) has a noticeably flatter $K$-factor than the ones for the two leading jets.

The di-photon invariant mass distribution in figure 9 (b) receives significant corrections to the shape at NLO with the $K$-factors increasing from around 1.0 at low $m_{\gamma \gamma}$ to 1.4 at large values of the photon pair invariant mass. In figure 10 we show the leading jet/leading photon separation $R_{\gamma_{1} j_{1}}$ and the azimuthal separation of the two leading jets $\Delta \phi_{j_{1} j_{2}}$. Both quantities receive large NLO corrections for small values of the observable, though notably not as large as the corresponding distributions in $p p \rightarrow \gamma \gamma+2 j$ where there is a more substantial increase in the available phase-space at NLO.

The PDF analysis was performed with the same set-up as for $p p \rightarrow \gamma \gamma+2 j$ with the four PDF sets compared at the same value of $\alpha_{s}\left(M_{Z}\right)=0.118$. Table 2 shows the results for the total cross section at the central scale $\mu_{R}=\widehat{H}_{T}^{\prime} / 2$. Again we see that PDF uncertainties for all sets are noticeably smaller than theoretical uncertainties estimated from scale variations and that central predictions from different sets differ by more than the nominal uncertainty obtained with each set. Figure 11 shows the comparison of the $m_{\gamma \gamma}$ distribution for the different PDF sets. The deviation between ABM11 and NNPDF, which again give the 


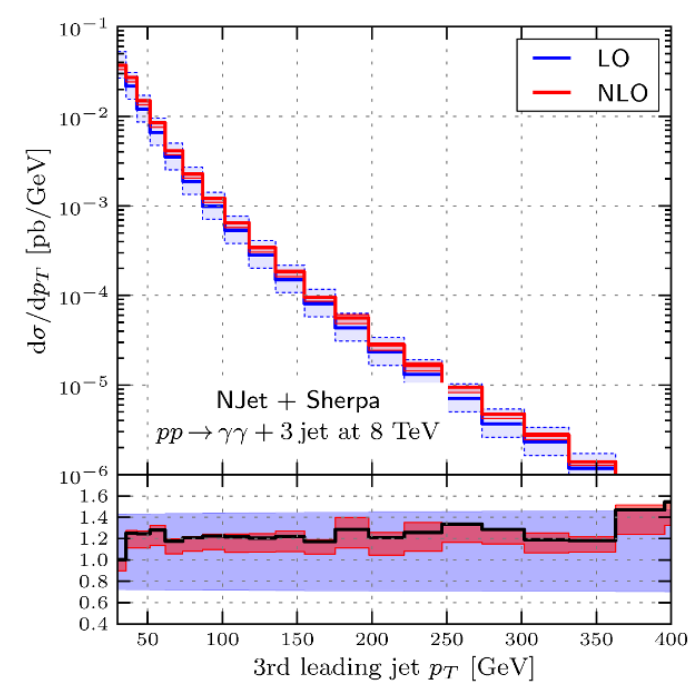

(a)

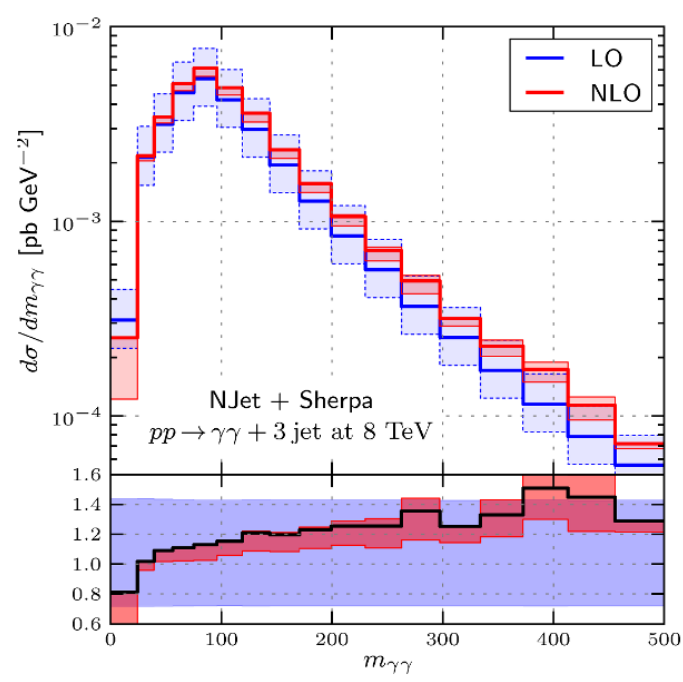

(b)

Figure 9. Differential cross section as a function of $p_{T}$ of the 3 rd leading jet and di-photon invariant mass in $p p \rightarrow \gamma \gamma+3 j$.
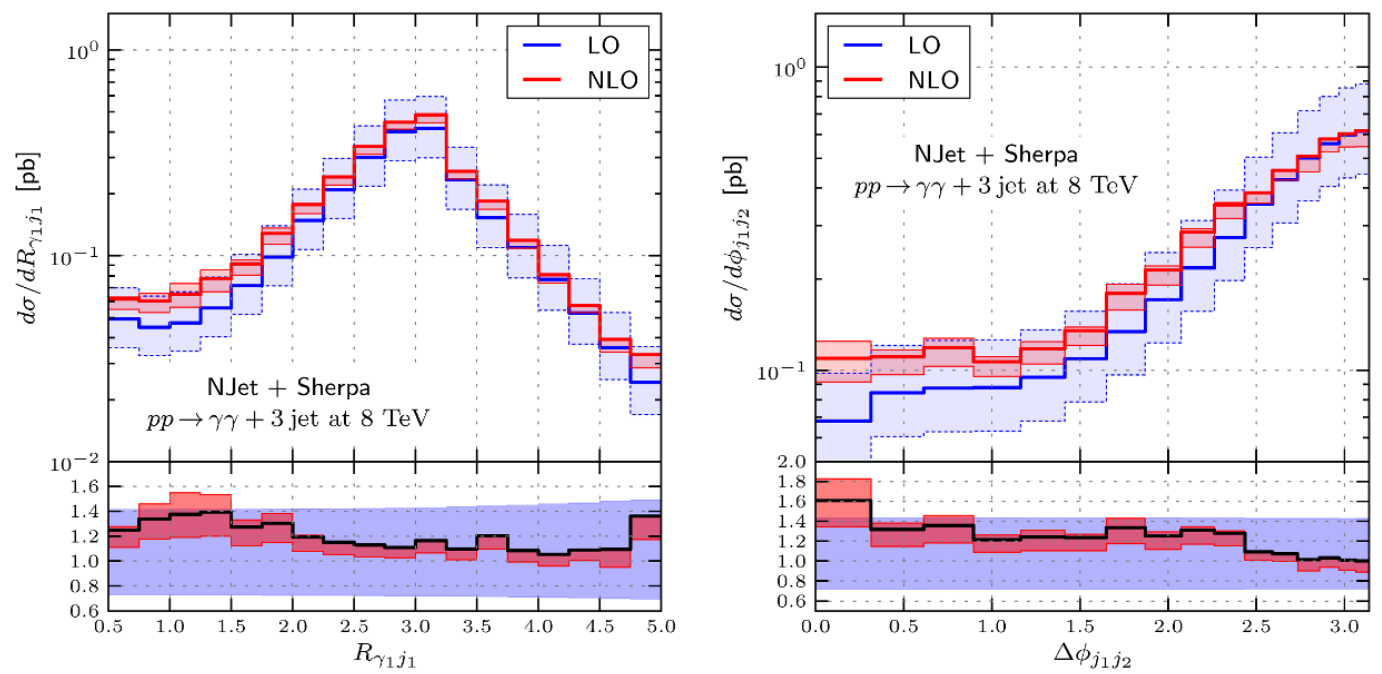

Figure 10. Photon/jet separation and di-jet azimuthal angle distributions in $p p \rightarrow \gamma \gamma+3 j$.

lower and higher limit of the predictions from different sets, is somewhat larger than that seen in $p p \rightarrow \gamma \gamma+2 j$, though the results are consistent within the theoretical uncertainties determined by scale variations.

\subsection{The three-to-two jet ratio}

In the context of multi-jet production studies it is interesting to look at the ratio of $p p \rightarrow$ $\gamma \gamma+3 j$ over $p p \rightarrow \gamma \gamma+2 j$ which we will denote as $R_{3 / 2}$. Due to the cancellation of many uncertainties both theoretical and experimental, ratios such as this one are prime observables for the determination of physical parameters (like $\alpha_{s}$ ). 


\begin{tabular}{|lccc|}
\hline PDF set & $\sigma_{\gamma \gamma+3 j}^{\mathrm{NLO}}\left(\widehat{H}_{T}^{\prime} / 2\right)$ & $\delta \sigma_{\gamma \gamma+3 j}^{\mathrm{NLO}, \mathrm{PDF}+}\left(\widehat{H}_{T}^{\prime} / 2\right)$ & $\delta \sigma_{\gamma \gamma+3 j}^{\mathrm{NLO}, \mathrm{PDF}-}\left(\widehat{H}_{T}^{\prime} / 2\right)$ \\
\hline CT10nlo & 0.746696 & +0.0123788 & -0.0133826 \\
NNPDF2.3 & 0.773112 & +0.0056425 & -0.0056425 \\
MSTW2008 & 0.752756 & +0.0068782 & -0.0050721 \\
ABM11 & 0.731019 & +0.0241568 & -0.0241568 \\
\hline
\end{tabular}

Table 2. The total cross section for $p p \rightarrow \gamma \gamma+3 j$ computed with different PDF sets at $\alpha_{s}\left(M_{Z}\right)=$ 0.118. The PDF uncertainties are computed from the relevant PDF error sets.

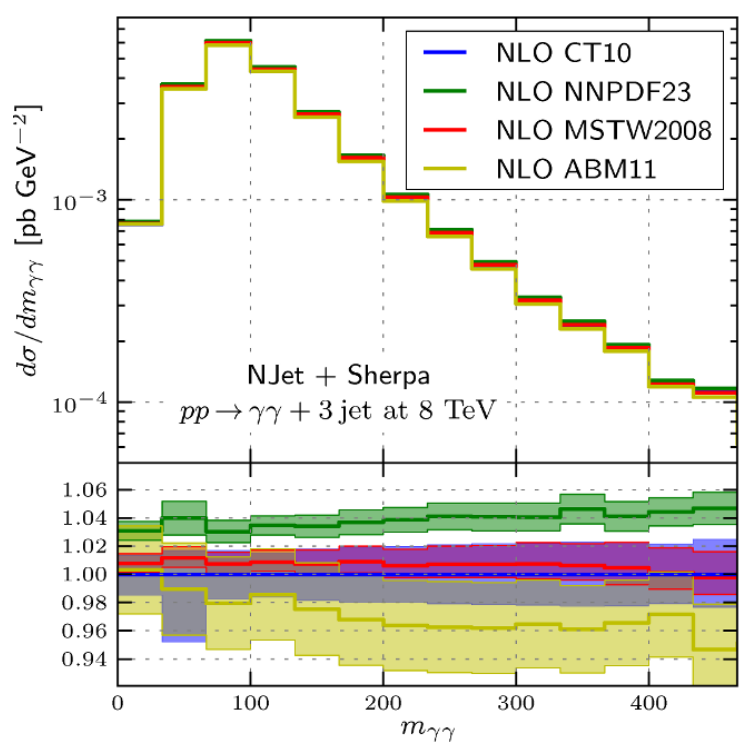

Figure 11. The $m_{\gamma \gamma}$ distributions for $p p \rightarrow \gamma \gamma+3 j$ for the four PDF sets described in the text at $\alpha_{s} M_{Z}=0.118$. The lower plot contains the ratio of each set to CT10 with the shaded region representing the PDF uncertainties.

In the case of the production of di-photon in association with jets, we find $R_{3 / 2}$ to be:

$$
R_{3 / 2}^{\mathrm{LO}}\left(\mu_{R}=\widehat{H}_{T}^{\prime} / 2\right)=0.314(0.002) \quad R_{3 / 2}^{\mathrm{NLO}}\left(\mu_{R}=\widehat{H}_{T}^{\prime} / 2\right)=0.276(0.004)
$$

where the numbers in brackets refer to Monte-Carlo errors. We have checked that all scale choices are in much better agreement for $R_{3 / 2}$ with the range of predictions lying within $\sim 8 \%$ of this value. In figure 12 we show the differential ratio with the $p_{T}$ of the leading jet. The NLO corrections become more important for $p_{T}>100 \mathrm{GeV}$ and reaching about $15 \%$ at high $p_{T}$.

\section{Conclusions}

In this paper we have presented a study of di-photon production in association with jets. The first calculation of the full NLO QCD corrections to the process $p p \rightarrow \gamma \gamma+3 j$ is 


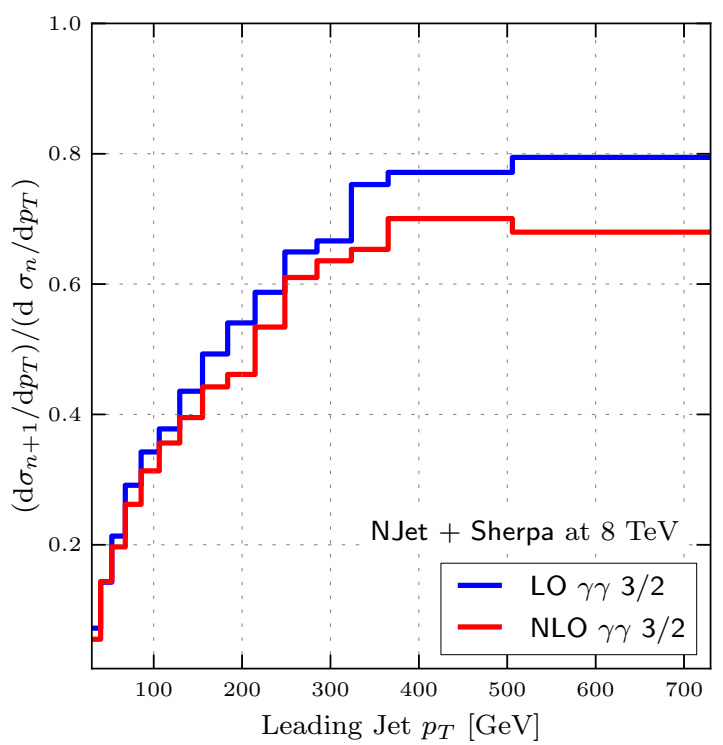

Figure 12. The ratio of of $p p \rightarrow \gamma \gamma+3 j$ over $p p \rightarrow \gamma \gamma+2 j$ as a function of leading jet $p_{T}$.

presented and discussed, together with a variety of results for $p p \rightarrow \gamma \gamma+2 j$. We find that the inclusion of NLO QCD corrections leads to a significant reduction of theoretical uncertainties both on total cross sections and distributions. We have studied distributions for a number of observables, of particular interest are those relevant for Higgs production in VBF analyses which are used when modelling $\gamma \gamma+$ jets as a background to $p p \rightarrow H+$ jets $\rightarrow$ $\gamma \gamma+$ jets.

The present study is based on the use of the Frixione smooth cone isolation criterion to define the final state photons, which provides a theoretically clean way of suppressing the fragmentation component in direct photon production. It would be also interesting to consider alternative isolation criteria, like the fixed cone isolation, which require the inclusion of fragmentation functions, and were shown to have a significant effect in lower multiplicity processes [7]. Moreover the majority of experimental analyses involving direct photon production (both in association with jets or not) rely on the cone isolation for photon identification. Nonetheless, we hope that the results presented here will be of use in future experimental analyses and look forward to direct comparisons with the LHC data.

\section{Acknowledgments}

We would like to thank Peter Uwer, Benedikt Biedermann, Thomas Gehrmann, Gudrun Heinrich and Nicolas Greiner and Nicolas Chanon for helpful discussions. We are grateful to the Humboldt-Universität zu Berlin for providing computing resources. This work has been supported by the Alexander von Humboldt Foundation, in the framework of the Sofja Kovaleskaja Award 2010, endowed by the German Federal Ministry of Education and Research.

Open Access. This article is distributed under the terms of the Creative Commons Attribution License (CC-BY 4.0), which permits any use, distribution and reproduction in any medium, provided the original author(s) and source are credited. 


\section{References}

[1] ATLAS collaboration, Observation of a new particle in the search for the standard model Higgs boson with the ATLAS detector at the LHC, Phys. Lett. B 716 (2012) 1 [arXiv: 1207.7214] [INSPIRE].

[2] CMS collaboration, Observation of a new boson at a mass of $125 \mathrm{GeV}$ with the CMS experiment at the LHC, Phys. Lett. B 716 (2012) 30 [arXiv:1207.7235] [INSPIRE].

[3] CMS collaboration, Rapidity distributions in exclusive $Z+$ jet and $\gamma+$ jet events in pp collisions at $\sqrt{s}=7$ TeV, Phys. Rev. D 88 (2013) 112009 [arXiv:1310.3082] [InSPIRE].

[4] ATLAS collaboration, Dynamics of isolated-photon plus jet production in pp collisions at $\sqrt{s}=7$ TeV with the ATLAS detector, Nucl. Phys. B 875 (2013) 483 [arXiv:1307.6795] [INSPIRE].

[5] S. Catani, L. Cieri, D. de Florian, G. Ferrera and M. Grazzini, Diphoton production at hadron colliders: a fully-differential QCD calculation at NNLO, Phys. Rev. Lett. 108 (2012) 072001 [arXiv: 1110.2375] [INSPIRE].

[6] V. Del Duca, F. Maltoni, Z. Nagy and Z. Trócsányi, QCD radiative corrections to prompt diphoton production in association with a jet at hadron colliders, JHEP 04 (2003) 059 [hep-ph/0303012] [INSPIRE].

[7] T. Gehrmann, N. Greiner and G. Heinrich, Photon isolation effects at NLO in $\gamma \gamma+$ jet final states in hadronic collisions, JHEP 06 (2013) 058 [arXiv: 1303.0824] [INSPIRE].

[8] S. Frixione, Isolated photons in perturbative QCD, Phys. Lett. B 429 (1998) 369 [hep-ph/9801442] [INSPIRE].

[9] T. Gehrmann, N. Greiner and G. Heinrich, Precise QCD predictions for the production of a photon pair in association with two jets, Phys. Rev. Lett. 111 (2013) 222002 [arXiv:1308.3660] [INSPIRE].

[10] Z. Bern et al., Next-to-leading order diphoton + 2-jet production at the LHC, arXiv: 1312.0592 [INSPIRE].

[11] Z. Bern, L.J. Dixon, D.C. Dunbar and D.A. Kosower, One loop $n$ point gauge theory amplitudes, unitarity and collinear limits, Nucl. Phys. B 425 (1994) 217 [hep-ph/9403226] [INSPIRE].

[12] R. Britto, F. Cachazo and B. Feng, Generalized unitarity and one-loop amplitudes in $N=4$ super-Yang-Mills, Nucl. Phys. B 725 (2005) 275 [hep-th/0412103] [INSPIRE].

[13] G. Ossola, C.G. Papadopoulos and R. Pittau, Reducing full one-loop amplitudes to scalar integrals at the integrand level, Nucl. Phys. B 763 (2007) 147 [hep-ph/0609007] [INSPIRE].

[14] C. Berger et al., Precise predictions for $W+4$ jet production at the Large Hadron Collider, Phys. Rev. Lett. 106 (2011) 092001 [arXiv:1009.2338] [INSPIRE].

[15] H. Ita et al., Precise predictions for $Z+4$ jets at hadron colliders, Phys. Rev. D 85 (2012) 031501 [arXiv: 1108.2229] [INSPIRE].

[16] Z. Bern, G. Diana, L. Dixon, F. Febres Cordero, S. Hoeche et al., Four-jet production at the Large Hadron Collider at next-to-leading order in QCD, Phys. Rev. Lett. 109 (2012) 042001 [arXiv:1112.3940] [INSPIRE]. 
[17] S. Badger, B. Biedermann, P. Uwer and V. Yundin, NLO QCD corrections to multi-jet production at the LHC with a centre-of-mass energy of $\sqrt{s}=8$ TeV, Phys. Lett. B 718 (2013) 965 [arXiv:1209.0098] [INSPIRE].

[18] Z. Bern et al., Next-to-leading order W+5-jet production at the LHC, Phys. Rev. D 88 (2013) 014025 [arXiv: 1304.1253] [INSPIRE].

[19] S. Badger, B. Biedermann, P. Uwer and V. Yundin, Next-to-leading order QCD corrections to five jet production at the LHC, Phys. Rev. D 89 (2014) 034019 [arXiv:1309.6585] [INSPIRE].

[20] C. Berger et al., An automated implementation of on-shell methods for one-loop amplitudes, Phys. Rev. D 78 (2008) 036003 [arXiv:0803.4180] [inSPIRE].

[21] V. Hirschi et al., Automation of one-loop QCD corrections, JHEP 05 (2011) 044 [arXiv: 1103.0621] [INSPIRE].

[22] G. Bevilacqua et al., HELAC-NLO, Comput. Phys. Commun. 184 (2013) 986 [arXiv:1110.1499] [INSPIRE].

[23] G. Cullen et al., Automated one-loop calculations with GoSam, Eur. Phys. J. C 72 (2012) 1889 [arXiv: 1111.2034] [INSPIRE].

[24] F. Cascioli, P. Maierhofer and S. Pozzorini, Scattering amplitudes with open loops, Phys. Rev. Lett. 108 (2012) 111601 [arXiv:1111.5206] [INSPIRE].

[25] S. Actis, A. Denner, L. Hofer, A. Scharf and S. Uccirati, Recursive generation of one-loop amplitudes in the standard model, JHEP 04 (2013) 037 [arXiv:1211.6316] [INSPIRE].

[26] F.A. Berends and W. Giele, Recursive calculations for processes with $n$ gluons, Nucl. Phys. B 306 (1988) 759 [INSPIRE].

[27] T. Gleisberg and S. Hoeche, Comix, a new matrix element generator, JHEP 12 (2008) 039 [arXiv:0808.3674] [INSPIRE].

[28] S. Catani and M. Seymour, The dipole formalism for the calculation of QCD jet cross-sections at next-to-leading order, Phys. Lett. B 378 (1996) 287 [hep-ph/9602277] [INSPIRE].

[29] T. Gleisberg and F. Krauss, Automating dipole subtraction for QCD NLO calculations, Eur. Phys. J. C 53 (2008) 501 [arXiv:0709.2881] [INSPIRE].

[30] T. Gleisberg et al., Event generation with SHERPA 1.1, JHEP 02 (2009) 007 [arXiv: 0811.4622] [INSPIRE].

[31] S. Badger, B. Biedermann, P. Uwer and V. Yundin, Numerical evaluation of virtual corrections to multi-jet production in massless QCD, Comput. Phys. Commun. 184 (2013) 1981 [arXiv: 1209.0100] [INSPIRE].

[32] S. Badger, B. Biedermann and P. Uwer, NGluon: a package to calculate one-loop multi-gluon amplitudes, Comput. Phys. Commun. 182 (2011) 1674 [arXiv:1011.2900] [InSPIRE].

[33] R.K. Ellis, W. Giele and Z. Kunszt, A numerical unitarity formalism for evaluating one-loop amplitudes, JHEP 03 (2008) 003 [arXiv:0708.2398] [inSPIRE].

[34] D. Forde, Direct extraction of one-loop integral coefficients, Phys. Rev. D 75 (2007) 125019 [arXiv:0704.1835] [INSPIRE].

[35] W.T. Giele, Z. Kunszt and K. Melnikov, Full one-loop amplitudes from tree amplitudes, JHEP 04 (2008) 049 [arXiv: 0801.2237] [INSPIRE]. 
[36] S. Badger, Direct extraction of one loop rational terms, JHEP 01 (2009) 049 [arXiv:0806.4600] [INSPIRE].

[37] T. Binoth et al., A proposal for a standard interface between Monte Carlo tools and one-loop programs, Comput. Phys. Commun. 181 (2010) 1612 [arXiv:1001.1307] [INSPIRE].

[38] S. Alioli et al., Update of the Binoth Les Houches Accord for a standard interface between Monte Carlo tools and one-loop programs, Comput. Phys. Commun. 185 (2014) 560 [arXiv: 1308.3462] [INSPIRE].

[39] M. Kretz and V. Lindenstruth, Vc: a C++ library for explicit vectorization, Software Pract. Exper. 42 (2012) 1409.

[40] R.K. Ellis, K. Melnikov and G. Zanderighi, Generalized unitarity at work: first NLO QCD results for hadronic $W+3$ jet production, JHEP 04 (2009) 077 [arXiv:0901.4101] [INSPIRE].

[41] SM and NLO Multileg Working Group collaboration, J. Andersen et al., The SM and NLO multileg working group: summary report, arXiv:1003.1241 [INSPIRE].

[42] T. Carli et al., A posteriori inclusion of parton density functions in NLO QCD final-state calculations at hadron colliders: the APPLGRID project, Eur. Phys. J. C 66 (2010) 503 [arXiv: 0911.2985] [INSPIRE].

[43] M. Cacciari, G.P. Salam and G. Soyez, The anti- $k_{t}$ jet clustering algorithm, JHEP 04 (2008) 063 [arXiv: 0802.1189] [INSPIRE].

[44] M. Cacciari, G.P. Salam and G. Soyez, FastJet user manual, Eur. Phys. J. C 72 (2012) 1896 [arXiv: 1111.6097] [INSPIRE].

[45] H.-L. Lai et al., New parton distributions for collider physics, Phys. Rev. D 82 (2010) 074024 [arXiv: 1007.2241] [INSPIRE].

[46] A. Martin, W. Stirling, R. Thorne and G. Watt, Parton distributions for the LHC, Eur. Phys. J. C 63 (2009) 189 [arXiv:0901.0002] [InSPIRE].

[47] S. Alekhin, J. Blumlein and S. Moch, Parton distribution functions and benchmark cross sections at NNLO, Phys. Rev. D 86 (2012) 054009 [arXiv: 1202.2281] [INSPIRE].

[48] R.D. Ball et al., Parton distributions with LHC data, Nucl. Phys. B 867 (2013) 244 [arXiv: 1207.1303] [INSPIRE]. 